\title{
Walther von der Vogelweide als \\ Vortragender \\ Das Mimische und die Strophenfolge seiner \\ Liebeslieder
}

\section{Rodney W. Fisher}

Den berühmten mittelalterlichen Minnesänger Walther von der Vogelweide für Österreich in Anspruch zu nehmen, dürfte den Laien kaum weniger abenteuerlich anmuten als etwa den Ötzi für Österreich beanspruchen zu wollen; gab es doch Ende des zwölften Jahrhunderts noch keinen Staat Österreich, nur das Herzogtum unter den Babenbergern, das selbst Teil des deutschen Reichs war. Außerdem war Walther bekanntlich 'ein deutscher Berufsdichter ohne festen Wohnsitz', 'ein armer Hund', 'einer, den Leben und Schicksal herumgestoßen und geschunden haben'.' Dabei hat es natürlich nicht an mehr oder weniger seriösen Versuchen gefehlt, die Heimat des Dichters zu lokalisieren oder gar aufzufinden, und zwar in Deutschland wie auch in Österreich, ${ }^{2}$ wohl zum Teil wegen des Prestiges, das mit seinem Namen verbunden war und ist, zum Teil aber auch, weil weder der Beiname 'von der Vogelweide' noch das Zeugnis seiner Lieder und Sprüche unzweideutige Schlüsse zuläßt. Auch die bekannte Aussage des Dichters selbst (ze Ôsterrîche lernt ich singen unde sagen $\mathrm{L} 19,29$ ) besagt nur, daß er vielleicht seine Jugendzeit im Herzogtum verbracht und da (wohl am Wiener Hof) die Kunst des Minnesangs gelernt hat. Die Aussage muß man aber auch vor dem Hintergrund einer gewissen literarischen Tradition sehen: Österreich war als bevorzugte Dichterheimat bekannt; außerdem ist es nicht ohne weiteres möglich, das Ich einer lyrischen Aussage mit einer bestimmten realen Person zu identifizieren ${ }^{3}$ Ferner steht einigermaßen fest; daß im

${ }^{1}$ Peter Wapnewski: Walther von der Vogelweide. Gedichte, Fischer Taschenbuch Verlag, Frankfurt a.M. 1973, S. 287ff.

${ }^{2}$ Als ein Beispiel unter vielen sei hier der Beitrag Alfred Krachers erwähnt: "Walthers Heimat: Franken oder Österreich?" in: Mediavvalia litteraria. Fs. f. Helmut de Boor, hrsg. von Ursula Hennig und Herbert Kolb, München 1971.

${ }^{3}$ Die Schwierigkeiten gerade bei Walther bezeugen eine Reihe neuerer 
Jahre 1198 Walther aus irgendeinem Grund (seine "Verdrängung" durch Reinmar, der das Amt eines "Wiener Hofpoeten" innegehabt haben soll, ist reine Hypothese) den Wiener Hof verließ, und daß er 1203 zum Gefolge des Passauer Bischofs Wolfger von Erla gehörte, der im OktoberNovember wegen der Hochzeit Leopolds VI. von Österreich in Wien war; ob Walther zu diesem Zeitpunkt auch in Wien war, wissen wir nicht, aber es ist zumindest möglich. Außer Zweifel steht auch, daß in Walthers Spruchdichtung Österreich und der Wiener Hof eine größere Rolle spielen als alle anderen Länder und Höfe, so daß man mit gewissem Recht von einer starken persönlichen Beziehung zu Österreich sprechen darf, ${ }^{4}$ wenn sich auch Walthers österreichische Herkunft nicht beweisen läßt.

In einem anderen Punkt ist die moderne Forschung auch etwas nüchterner geworden. Lange Zeit galt Walther als ein Minnesänger mit besonders kräftigem Selbstbewußtsein, nachdem konstatiert wurde, daß in seiner Dichtung das Pronomen 'ich' das häufigste Wort sei. ${ }^{5}$ Zweifellos spielte hier eine weitere Walther-Legende mit, das Bild des großen Erneuerers, der sich gegen eine erstarrte Tradition aufgebäumt und der Kunst des Minnesangs seinen persönlichen Stempel aufgedrückt haben soll. So neu waren aber die meisten Gedanken Walthers nicht, und auch das angeblich starke Hervortreten seiner Persönlichkeit, die durch die Statistik der Ichaussagen bewiesen sein soll, läßt sich bei näherer Untersuchung des Materials nicht mehr aufrechterhalten. In seinen Minnesang-Strophen kommt das Ich nur geringfügig häufiger vor als bei den meisten Dichtern von Minnesangs Frühling, und sogar weniger häufig als bei seinen großen Zeitgenossen Morungen und Reinmar. ${ }^{6}$ Wenn Walthers Lieder

Veröffentlichungen zum Problem des "lyrischen Ichs", einige davon in den beiden Bänden Walther von der Vogelweide: Beiträge zu Leben und Werk (Günther Schweikle zum 60. Geburtstag), hrsg. von Hans-Dieter Mück, Stuttgart 1989, und Walther von der Vogelweide. Hamburger Kolloquium 1988 zum 65. Geburtstag von Karl-Heinz Borck, hrsg. von Jan-Dirk Müller und Franz Josef Worstbrock, Stuttgart 1989. Um der Klarheit willen verweise ich im folgenden auf Fs. Schweikle bzw. Fs. Borck. Was den Minnesang im allgemeinen betrifft, s. auch Jan-Dirk Müller: "'Ir sult sprechen willekommen.' Sänger, Sprecherrolle und die Anfänge volkssprachiger Lyrik" in: IASL 19 (1994), S. 1-21 (mit weiterer Literatur).

${ }^{4}$ Günther Schweikle: 'Walther und Wien. Überlegungen zur Biographie' in: Fs. Schweikle, S. 75-87.

5"Kein anderer mittelhochdeutscher Lyriker sagt so oft: Ich" (Joerg Schaefer in: Walther von der Vogelweide. Werke, Darmstadt 1972, S. XIII). 
trotzdem lebendig wirken, ist das vielleicht auf die Art und Weise zurückzuführen, wie sich dieses Ich artikuliert.

Beim mittelalterlichen Lied handelt es sich aber nicht nur um ein Ich, sondern auch - und zwar in weit konkreterem Maße als bei der modernen Leselyrik - um ein Du. Die Lieder wurden vor einem Publikum gesungen, entweder vom Dichter selbst oder von einem anderen, und das Ganze war teilweise gleichzeitig ein kleines Drama. Als Text konnten sie natürlich auch an eine Dame geschickt werden, ja, man kann annehmen, daß Frauen meist den geschriebenen Text besser zu schätzen wußten als Männer, die im allgemeinen weniger Bildung hatten und in den meisten Fällen nicht lesen konnten. Beim realen Vortrag aber, am Hof, eventuell unter musikalischer Begleitung, mußte man mit einem Publikum von recht unterschiedlicher Bildung rechnen, von den litterati mit Kenntnissen von Texten verschiedenster Art, bis zu den völlig illitterati. ${ }^{7}$

Nach dem Zeugnis von epischen Texten konnten Lieder direkt vor einer bestimmten Frau vorgetragen werden: so spielt und singt im Nibelungenlied der videlaere Volker seine Lieder direkt vor Gotelinde $(1705,1)$, während Gottfrieds Tristan für "seine" Isolde Lieder komponiert, die er aber vor der falschen Isolde, Isolde Weißhand, vorträgt (1920ff.). ${ }^{8}$ Zwar möchte man aus literarischen Texten nicht ohne weiteres auf die reale Praxis schließen, aber gegen das traditionelle Bild des Minnesängers, der seine Lieder vor Damen in der Gesellschaft singt, läßt sich wohl nichts einwenden. Wenn Walther sagt: Ich sanc hie vor den frowen umbe ir blôzen gruoz (L 49, 12), meint er eben, daß er vor Frauen am Hof gestanden und seine Liebeslieder vorgesungen hat (allerdings ist eine andere Deutung des Verses möglich; dazu s. unten). Vielleicht war es sogar eher vom Minnesang als von den Ritterromanen, daß Frauen sich "angesprochen" fühlten. Von einer weiteren Hypothese, die freilich eben-

${ }^{6}$ Diese Neubewertung der Statistik bei Joachim Knape: "Rolle und lyrisches Ich bei Walther" in: Fs. Schweikle, S. 171-190.

${ }^{7}$ Zur Komposition des Publikums s. den Überblick über neuere Forschung bei D. H. Green: "Vrume rîtr und guote vrouwen/und wîse phaffen. Court Literature and its Audience" in: German Narrative Literature of the Twelfth and Thirteenth Centuries, hrsg. Volker Honemann u.a., Tübingen 1994, S. 7-26; allerdings berücksichtigt Green nur das Publikum bei erzählenden Dichtungen.

${ }^{8}$ Diese und weitere Beispiele bespricht Joachim Bumke: Höfische Kultur, München 1987, S. $751 \mathrm{ff}$. 
so unbewiesen bleiben muß, gehe ich im folgenden aus: zwischen verschiedenen Gruppen am Hof konnte sich der Minnesänger während seines Vortrags relativ frei bewegen, konnte seine Worte mal an die eine, mal an die andere Gruppe richten. Dies findet seinen Niederschlag in der Anredeform ir bzw. $d u$, die gelegentlich, aber nicht immer, einen bestimmten Adressaten oder eine bestimmte Adressatengruppe meint. Umgekehrt konnte auch eine bestimmte Person oder Gruppe gemeint sein, oder sich persönlich angesprochen fühlen, obwohl der Vortragende ein Pronomen der dritten Person gebraucht; bekanntlich richtet der Minnesänger häufig seine Klage oder sein Lob an eine bestimmte Person, auf die er sich aber mit si bezieht, wobei natürlich nicht auszuschließen ist, $\mathrm{daß}$ die Angesprochene persönlich anwesend ist. Ähnliches gilt für das pluralische si, wenn Gruppen wie die zwivelaere $(\mathrm{L} \mathrm{58,21)}$ die lïgenaere (L 44, 23), die schamelosen ( $\mathrm{L} 64,4)$ gemeint sind; mit einer Geste oder einem Blick auf die Betroffenen oder dadurch, daß er sich ihnen zu- oder von ihnen abwandte, hătte der Minnesänger die Möglichkeit, die Scheinwerfer auf sie zu richten. Eine Beziehung zum Publikum konnte man natürlich auch durch das Pronomen wir herstellen, oder indem man mit einer rhetorischen Frage anfing, wie in Walthers bekanntem Lied Saget mir ieman, waz ist minne? (L 69, 1). Auch wenn die Lieder höchstwahrscheinlich als Solos vorgetragen wurden, hätte der Minnesänger durch die eigene Stellung dem Publikum gegenüber einen fingierten Dialog inszenieren können.

Der Wechsel vom si zum $d u$ oder $i r$, mitten im Lied oder sogar mitten in einer Strophe, zeugt von einer ständigen Spannung beim Vortrag, wohl auch von der Möglichkeit einer Bewegung seitens des Vortragenden, der nicht nur als Sänger sondern auch als Schauspieler auftritt. Das lyrische $\mathrm{Du}$ ist aber kaum weniger problematisch als das lyrische Ich. Von einem realen Verhältnis zwischen dem Minnesänger und einer bestimmten Frau kann keine Rede sein, oder besser: ein Verhältnis kann kein Thema für seriöse Forschung sein. Was am Hof als Minnesang vorgespielt wird, ist eben ein Spiel, mit entsprechenden Konventionen. Das heißt allerdings nicht, daß eine bestimmte Dame nicht als Adressat fungieren kann. Der Adressat erscheint manchmal auch als die Personifizierung von Abstraktionen. Wenn Walther frowe Mâze, fro Welt, fro Unfuoge, fro Staete, her Meie (in dem bekannten Mailied L 45, 37), her 
Stoc (einen Opferstock), hovelichez singen (den höfischen Minnesang L 64, 31), oder auch Frowe Minne apostrophiert, so ist ein reales Gegenüber wohl auszuschließen, obwohl im Falle von Frowe Minne eine bestimmte Dame als Verkörperung der Minne denkbar wäre. Gerade in seinem äußerst häufigen Gebrauch der Apostrophe zeigt sich meines Erachtens Walthers Hang zum Dramatischen.

Vor einigen Jahren hatte Wolfgang Mohr gemeint, die Dame in Walthers Minnesang könnte unter Umständen allegorisch verstanden werden, als Verkörperung des Hofes. ${ }^{9}$ Auch wenn das für das Du bzw. Ihr der Anrede gelten sollte, würde es nicht ausschließen, daß gleichzeitig auch eine bestimmte Frau gemeint ist oder angesprochen wird, d.h. daß das Lied eine "persönliche" wie auch eine gesellschaftliche Botschaft enthält. Bei einigen programmatischen Strophen, in denen ein Teil des Publikums mit ir angesprochen wird, ist an der Direktheit der Botschaft und an der Anwesenheit der Adressaten kaum zu zweifeln; so die Ritter, die in der dritten Strophe von Walthers sog. Elegie (L 125, 1ff) aufgefordert werden, am Kreuzzug teilzunehmen. Hier müßte man allerdings zwischen den Liebesliedern und den Sprüchen unterscheiden; wo letztere an einen mächtigen Fürsten oder gar an den Kaiser gerichtet werden, braucht man natürlich nicht mit der Gegenwart des Adressaten zu rechnen.

Auch in den Liebesliedern braucht nicht jede Du- oder Ihrform als direkte Anrede verstanden $\mathrm{zu}$ werden. Hier denke ich an Lieder wie Walthers Botenlied (L 112, 35), sein Tagelied Friuntlichen lac (L 88, 9) oder sein Lied von der Traumliebe Nemt, frowe, disen kranz (L 74, 20); die beiden letzteren enthalten starke epische Elemente, wozu auch der vergegenwärtigte Dialog gehört, der aber als kleine Inszenierung schwer vorstellbar ist. Über die Art des Vortrags wissen wir wenig, ob oder wie der Minnesänger eine Frauenstimme signalisieren konnte, ist unbekannt. Echte Dialoglieder, d.h. als reine Gespräche zwischen Minnepartnern konzipierte Lieder, mit oder ohne narrative Verbindungen, finden sich erst im dreizehnten Jahrhundert häufiger. ${ }^{10}$ In Walthers Werk gibt es nur drei

${ }^{9}$ Der Gedanke wurde dann von anderen weiterentwickelt; s. Jan-Dirk Müller: "Die Frouwe und die anderen. Beobachtungen zur Überlieferung einiger Lieder Walthers" in: Fs. Borck, S. 127-146.

${ }^{10}$ Zum Dialoglied Theodor Frings: "Walthers Gespräche" in: Walther von der Vogelweide, hrsg. S. Beyschlag, Darmstadt 1971, S. 420-430 und Ingrid Kasten: "Das Dialoglied bei Walther von der Vogelweide" in: $F s$. Borck, S. 81-94, wo man liest, daß "Walther von der Vogelweide der erste 
Beispiele (L 85,34; L 43, 9; L 70, 22), bei denen das Gespräch strophenweise wechselt (nur in letzterem fällt eine Strophe, sozusagen beiseite gesprochen, aus dem Schema). Hinzu kommen allerdings die vielen Lieder Walthers, in denen eine allegorische Gestalt als Dialogpartner erscheint und ein bestimmter Adressat unter dem Publikum kaum vorstellbar ist.

Von größerer Relevanz bei der Frage der Wechselbeziehung zwischen Walther und seinem Publikum sind natürlich die gelegentlichen Du- und Ihrformen innerhalb eines Liedes. Von den rund zweihundertsechzig Strophen, die in der Ausgabe von Friedrich Maurer die Liebeslieder ausmachen ${ }^{11}$ finden sich etwa $10 \%$, in denen das Publikum oder ein Teil davon mit ir angesprochen wird. Dabei handelt es sich ein paar mal nur um die Verbform seht, die wohl schon als Interjektionspartikel fungieren konnte (mit der Bedeutung etwa sieh da!), in vielen Fällen aber ist an einer echten Anrede nicht zu zweifeln, z.B. wenn bestimmte Adressaten genannt werden: nu ratet, friunt, waz ich es tuo (innerhalb einer Frowe-Minne-Apostrophe, L 55,37); ir sult si miden, guotiu wip (in einer Besprechung der Entsagung, L 41, 13); nu hat si [ = ein vil wunderaltez wip] mir bescheiden / waz der troum bediute,/ daz merket, lieben liute! (in seinem Lied vom Traumglück, L 94, 11). Bei dieser Statistik des "Publikums-Ihr" muß allerdings wieder betont werden, daß man an keine reale Teilnahme seitens der Gesellschaft zu denken braucht. Das direkte Werbe- oder Klagelied, d.h. Minnesang in Form einer direkten Umwerbung mit $d u$ bzw. ir, auch "Anredelied" genannt, ist auch relativ selten. Der Germanist Günther Schweikle, der in seinem Buch Minnesang über Wesen und Form der Gattung referiert und dessen Ergebnisse ich mir hier zu eigen mache, ${ }^{12}$ findet knapp ein Dutzend Beispiele für Anredelieder vor Walther, und bei Walther selbst nur drei. Vereinzelte Anredeformen innerhalb eines Liedes oder einer Strophe scheint Schweikle übersehen zu haben, und einige Lieder, die er nicht berücksichtigt, könnten ebensogut als Anredelieder gelten, aber an seiner

Minnesänger ist, der reine Dialoglieder komponiert hat" (S. 81). Auch das legt nahe, wie gerne Walther 'dramatisiert'.

${ }^{11}$ Die Lieder Walthers von der Vogelweide. 2. Bändchen: Die Liebeslieder, Tübingen (ATB) 1962. Nach dieser Ausgabe wird im folgenden zitiert, allerdings unter Beibehaltung der Numerierung Lachmanns.

${ }^{12}$ Minnesang, Stuttgart (Metzler) 1989, S. 122ff. 
Klassifikation der Gattung ändert das natürlich nichts. Er konstatiert drei Merkmale von Anredeliedern, und zwar (1) ihre relative Seltenheit, (2) die Gleichsetzung von frouwe und wîp als Gesprächspartner des lyrischen Ichs, und (3) die Tatsache, daß Inhalt und Modus der Anredelieder den Minneklagen ohne Adressierung entsprechen, d.h. Topoi und Motive der Anredelieder unterscheiden sich nicht von denen im sonstigen Minnesang. Auch Schweikles Schlußfolgerung sei hier zitiert: "Diese Momente [...] sind deutliche Belege dafür, daß auch die scheinbar konkreter auf bestimmte Frauen bezogenen Anredelieder nur Rollenspiel, nur eine formale Variante ein- und derselben lyrischen Fiktion ist". Wenn man erst einmal von der etwas unklaren Logik absieht - wieso gerade diese Merkmale beweisen sollen, daß auch Anredelieder nur Rollenspiel seien, ist mir nicht ersichtlich - kann man Schweikles Schluß insofern akzeptieren, als keine Minnelieder reale Beziehungen zwischen einem Minnesänger und "seiner" Dame darstellen. Daß es trotzdem diese - wie auch seltene - Sonderform des Anredeliedes gibt, scheint mir eher ein Beweis dafür zu sein, daß die Minnesänger gelegentlich ein fingiertes Verhältnis in dramatischerer Form darstellen wollten, als es die anderen Formen des Minnesangs zuließen. Ob der Minnesänger als Form das Anredelied wählte, hatte wahrscheinlich nichts mit dem Inhalt zu tun (da alle Beziehungen zu Umworbenen sowieso zur Konvention gehörten); man hatte sich wohl eher vom jeweiligen Publikum leiten lassen: wie würde eine bestimmte Frau bzw. eine Gruppe am Hof darauf reagieren, wenn der Minnesänger sich direkt vor sie stellte? ${ }^{13}$

Nach dem Zeugnis seiner Dialoglieder, seiner Anredelieder, und seiner Duund Ihr-Anreden scheint Walther in dieser Hinsicht weniger zurückhaltend gewesen zu sein als seine Vorgänger. Ferner, in seinen vielen Apostrophen (fingierten Dialogen mit fro Staete, fro Maze usw.) bestätigt sich sein Hang zum Dramatischen. Diese Erkenntnisse können wir guten Gewissens bei der Interpretation seiner Lieder verwerten, indem wir uns gewisse Bewegungen und Gesten vorstellen, die den Vortrag begleitet haben können. Freilich kann eine solche Rekonstruktion nur eine Hypo-

${ }^{13}$ Natürlich sind direkte Anreden sowie Dialoge 'fingiert' (s. den Versuch einer Typologie bei Christoph Cormeau: "Versuch über typische Formen des Liedeingangs bei Walther" in: Fs. Borck, S. 115-126, bes. S. 118f.); das schließt aber nicht aus, daß der Sänger eine bestimmte Person als Gegenüber suchte. 
these sein, eine Hypothese, die außerdem gelegentlich auf einer bestimmten Strophenfolge basiert. Die neueste Forschung zum Minnesang hat uns gelehrt, daß man bei der Edition mittelhochdeutscher Texte mit den überlieferten Handschriften viel konservativer umzugehen hat als vor hundert oder noch vor dreißig Jahren der Fall war, daß die handschriftlichen "Varianten" keine mehr oder weniger verläßlichen Abweichungen von irgendwelcher Norm darstellen können, sondern eben Variationen waren, bei denen der Dichter selbst beteiligt gewesen sein kann. "Vor wechselndem Publikum konnte ein Text variabel bleiben, konnte der jeweiligen Stimmung, Interessenlage, Aufnahmebereitschaft angeglichen werden". ${ }^{14}$ Wo ein Lied in verschiedenen Gestalten überliefert ist, mit verschiedenem Strophenbestand oder verschiedener Strophenfolge, haben wir es vielleicht mit einer Reihe von Aufführungen zu tun, die verschiedentlich aufgezeichnet wurden. So wie im Text, hätte sich der Minnesänger auch in seiner Vortragsweise der jeweiligen Situation anpassen können, d.h. eine Strophe, die ein Gegenüberstehen erforderte oder nahelegte, konnte einfach beliebig weggelassen oder hinzugefügt, oder anders geordnet werden. ${ }^{15}$

Ein Beispiel für die verschiedenen Möglichkeiten bietet L 69, 1 Saget mir ieman, waz ist minne, ein Lied, das wegen seiner vermeintlich programmatischen Aussagen zu den berühmtesten in Walthers Corpus gehört. ${ }^{16}$ In der Strophenfolge Lachmanns, der auch Maurer folgt und die durch drei der sechs Handschriften bezeugt wird, beginnt das Lied mit einer rhetorischen Frage, die der Minnesänger an sein Publikum als ganzes richtet. Darauf folgt eine Herausforderung, die gleichermaßen das

${ }^{14}$ Schweikle (Anm. 12), S. 27; vgl. auch verschiedene Aufsätze Schweikles in seinem Minnesang in neuer Sicht, Stuttgart, Weimar 1994. Eine ganz andere Meinung vertritt Hans Günther Meyer: Die Strophenfolge und ihre Gesetzmäßigkeiten im Minnelied Walthers von der Vogelweide (Deutsche Studien Bd. 35), Königstein/Ts. 1981, S. 526. Es versteht sich von selbst, daß bei seiner Erörterung der hier behandelten Lieder Meyer jeweils nur $e$ in $e$ Strophenfolge berücksichtigt.

${ }^{15}$ Zum mangelnden Fortschritt der Editionspraxis in Sachen Strophenfolge s. jetzt Manfred Günter Scholz: "Probleme der Strophenfolge in Walthers Dichtung" in: Fs. Schweikle, S. 207-220.

${ }^{16}$ Eine Relativisierung der herkömmlichen Meinung bot schon Silvia Ranawake: "Gab es eine Reinmar-Fehde?" in: Oxford German Studies 13 (1982) S. 7-35, S. 13ff. Zur Strophenfolge in diesem Lied s. Scholz (Anm. 15), S. $216 \mathrm{ff}$. 
Publikum einbezieht, und die erste Strophe mündet dann in einer Ichaussage des Minnesängers. Strophe 2 ist ähnlich gebaut: auf eine ans Publikum gerichtete Bitte um Bestätigung seiner Kompetenz in Sachen Liebe, die vielleicht mit einem einladenden Blick oder einer Geste begleitet wurde, folgt die etwas breiter ausgeführte Eigenmeinung mit der bekannten "Definition" Minne ist zweier herzen wünne. Ganz am Ende der Strophe scheint der Sänger dann plötzlich vor einer bestimmten Frau stehen zu bleiben, an die er seinen Aufruf richtet: das betonte "Du" unterbricht die sachliche Analyse und sollte wohl als Schock wirken, obwohl es doch folgerichtig und logisch aus der Definition (zweier herzen) hervorgeht. Während der dritten Strophe versucht er, das Überraschungsmoment voll auszunützen, indem er vor der Frau seine persönliche dringende Bitte fortsetzt und sich zu einer versteckten Drohung hinreißen läßt: weist sie ihn ab, so muß sie auch mit gewissen Konsequenzen rechnen. In der vierten Strophe reagiert der Sänger, als ob er in ihrem Blick oder einer Geste eben diese Abweisung gesehen hätte. Jetzt spricht er, vielleicht teilweise wieder den anderen Zuhörern zugewandt, von ihr in der dritten Person, während er an die Vernunft und das Gerechtigkeitsgefühl des Publikums appelliert, als ob die Dame Gegenstand einer öffentlichen Anklage wäre. In der letzten Zeile aber kommt die revocatio, wenn der Sänger vor der Macht der Liebe resigniert und sich ganz von der Dame abwendet, oder vielleicht sozusagen "die Bühne verläßt".

Ein ganz anderes Drama aber ergibt sich aus der Strophenfolge, wie sie in Hs. A überliefert ist. Hier fängt das Lied mit der persönlichen Konfrontation an (Strophe 3); die Mahnung der letzten Zeile scheint trotzdem genausowenig Erfolg zu haben, da sie wieder in die kurzlebige Entrüstung von Strophe 4 führt, jetzt mit dem ersten Versuch, die anderen am Hof auf die Seite des Sängers zu bringen. Die Resignation der letzten Zeile geht dann Hand in Hand mit einer Bewegung auf die anderen zu (durch die rhetorische Frage angeregt?) und vor ihnen appelliert er an den Gerechtigkeitssinn des Hofes während der Definition der Liebe in Strophe 2. Die berühmte Aussage Minne ist zweier herzen wünne würde dann aus der persönlichen, erfolglosen Konfrontation resultieren, wäre eine Einsicht, zu der man aus bitterer Erfahrung gelangt. Die ersten Zeilen, die eine Anerkennung durch das Publikum suchen, hätten einen leicht ironischen Ton, die letzte Halbzeile könnte mit einem letzten resignierten Blick 
zurück auf die Frau gesungen werden. Als Schluß des Liedes bleibt Strophe 1; hier wird die Unbegreiflichkeit des Phänomens Minne nicht als These eingeführt, die noch zu testen ist, sondern als Zusammenfassung der ganzen Aufführung. Die letzte Zeile bestätigt nur, was der Vortrag live gelehrt hat.

Eine dritte Version bietet Hs. C, die der Strophenfolge von Lachmann folgt, aber mit Strophe 4 an erster Stelle. Damit würde die Dame zum ersten Thema der Überlegungen, ohne daß sie direkt angesprochen wird. Die ans Publikum gerichteten rhetorischen Fragen, die am Schluß der Strophe den Sänger als einen "Unzurechnungsfähigen" disqualifizieren, sollen den teils ironischen Rahmen bilden für weitere Diskussionen mit dem Publikum, während deren er sich aber allmählich als seriöser Analytiker der Minne entpuppt. Dann kommt die plötzliche Wendung zur Dame hin, mit dem herausfordernden "Du", und dem ihr vom Sänger selbst gestellten Ultimatum der dritten Strophe (Z. 3-4). Die Spannung des ganzen Liedes gipfelt in der Drohung der letzten Zeile. In dieser Aufführung bildet die persönliche Konfrontation eine Probe auf die Richtigkeit der These minne ist zweier herzen wünne; das Publikum wartet gespannt auf das Ergebnis.

Außer den vier hier besprochenen Strophen gibt es aber eine fünfte, die in den meisten Ausgaben nicht gedruckt wird, die aber in drei Hss. überliefert ist, und zwar als vorletzte ( $\mathrm{L} 190,1)$ :

Ich wil also singen immer, daz si danne sprechent er ensanc nie baz.

Des gedankest du mir nimmer: daz verwise ich dir alrest, so denne daz. Weistu wie sie wünschent dir? daz si saelic si, durch di man uns sus singet. sich, frouwe, den gemeinen wunsch hast ouch von mir.

Die intensive Du-Anrede umfaßt Sänger und Dame als Partner in einem Sondergespräch innerhalb des größeren Publikums, auf das aber gelegentlich hingedeutet wird (si Z. 2, 5), und zu dem letzten Endes auch die Partner gehören (uns Z. 6). Die Strophe paßt also gut nach der Konfrontation in Strophe 3 mit ihrer versteckten Drohung am Schluß, aber vor 
der Enttäuschung und Abkehr der Strophe 4; die Zusatzstrophe enthält Kritik (wie auch immer der zweite Stollen, speziell Z. 4, zu interpretieren ist) ${ }^{17}$ bietet dem Sänger aber auch eine letzte Gelegenheit, intensiven Druck auszuüben, indem er vielleicht mit einem Blick oder einer Geste auf die anderen Zuhörer hindeutet (sich, frouwe), auf die die Dame auch Rücksicht nehmen soll. Erst als dieser Versuch scheitert, kehrt er ihr den Rücken und schließt sich wieder den anderen an (Strophe 4).

Bei einem anderen berühmten Lied, das auch zu den "programmatischen" gehört, können wir an verschiedene Darstellungsformen denken, zumal die Strophenfolge und der Strophenbestand in fünf Hss. jeweils anders überliefert sind. Es handelt sich um L 47, 36 Zwo fuoge han ich doch, swie ungefüege ich si, in dem sich Walther mit Gedanken Reinmars auseinandersetzt. Hier ist nicht der Platz, eine detaillierte Analyse der verschiedenen Möglichkeiten zu unternehmen, die eigentlich Aufgabe der Textkritik ist. Das Lied ist für meine Fragestellung nur bedingt relevant; die meisten Verse sind reine Argumentation, es kommt nicht zur Konfrontation mit einem $\mathrm{Du}$, und Bewegungen seitens des Vortragenden kann man sich kaum vorstellen. In zwei Strophen aber, die in drei von fünf Hss. aufeinander folgen, scheint der Sänger zwei verschiedene Gruppen direkt anzureden:

3 Ich sage iu waz uns den gemeinen schaden tuot: diu wip gelichent uns ein teil ze sere.

Daz wir in also liep sin übel alse guot, seht, daz gelichen nimet uns fröide und ere.

Schieden uns diu wip als e, daz si sich ouch liezen scheiden!

Daz gefrumt uns iemer me, mannen unde wiben beiden.

Waz stet übel, waz stet wol, sit man uns niht scheiden sol?

${ }^{17}$ Die Wendung alrerst so denne daz, die dunkel bleibt, begegnet sonst nur in einer anderen Strophe Walthers, die als unecht gilt (L 176,1; s.u.), hier interessanterweise auch innerhalb einer Du-Anrede; vielleicht gehörte der Ausdruck der Umgangssprache an. Auch dieses Lied enthält aber wichtige Aussagen über das Wesen der Minne, darunter die bekannte Definition, die sich teilweise mit Gedanken in L 69, 1 deckt: Minne entouc niht eine,/ si sol sin gemeine/so gemeine daz si ge/ dur zwei herze und dur dekeinez me. 
Edeliu wip gedenket

daz och die man waz kunnen:

gelichents iuch, ir sit gekrenket.

4. Wip muoz iemer sin der wibe hohste name, und tiuret baz dan frowe, als ichz erkenne.

Swa nu deheiniu si diu sich ir wipheit schame, diu merke disen sanc und kiese denne.

Under frowen sint unwip, under wiben sint si tiure. wibes name und wibes lip die sint beide vil gehiure.

Swiez umb alle frowen var, wip sin alle frowen gar! Zwivellop daz hoenet, als under wilen frouwe: wip dest ein name ders alle kroenet.

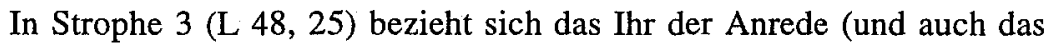
uns) anscheinend auf die Männer, da in der nächsten Zeile von diu wip die Rede ist, die als Gruppe Gegenstand der Kritik sind. So bleibt es dann bis Zeile 10, allerdings mit einer gewissen Auflockerung in der Mitte der Strophe, wo das "uns" eine gemischte Gruppe aus mannen unde wiben beiden meint; beide Geschlechter sind betroffen, wenn Frauen nicht mehr differenzieren. Während des Vortrags stellt sich der Sänger vielleicht vor eine neue Gruppe. Mit Z. 11 aber richtet er seine Worte deutlich an ein drittes Ihr, die adligen Damen: edeliu wip gedenket/daz och die man waz kunnen:/ gelichents iuch, ir sit gekrenket. Da hier auch eine ähnliche Drohung zum Ausdruck kommt wie in L 69, 1, sollte diese Bewegung auf die Frauen zu wahrscheinlich wieder als Schock wirken.

In allen drei Hss., die sowohl diese Strophe wie auch L 48, 38 (Strophe 4) enthalten, erscheinen die Strophen in der Lachmannschen Reihenfolge, d.h. es existierte eine gewisse Tradition, daß Walthers berühmte Strophe Wip muoz iemer sin der wibe hohste name direkt auf L 48, 25 folgen sollte. Mit gutem Grund, da am Ende von L 48, 25 der Sänger direkt vor den Damen steht, die sich doch von seinen Bemerkungen über die beiden 
Begriffe wip und frowe betroffen fühlen mußten. Bei Lachmanns letzter Strophe ( $\mathrm{L} 49,12$ Ich sanc hie vor den frowen umbe ir blozen gruoz), in der der Sänger seine Ungeduld in ungewöhnlich direkter Weise klarmacht, und die man sich nach ihrer ersten Zeile wohl als vor Frauen gesungen vorstellen könnte, ist die Lage nicht ganz so eindeutig, da die Strophe in einer der drei sie enthaltenden Hss. vor L 48, 25 erscheint:

5 Ich sanc hie vor den frowen umbe ir blozen gruoz, den nam ich wider mime lobe ze lone.

Swa ich des geltes nu vergebene warten muoz

da lobe ein ander, den si grüezen schone.

Swa ich niht verdienen kan

einen gruoz mit mime sange,

dar kere ich vil herscher man

minen nac ode ein min wange.

Daz kit "mir ist umbe dich

rehte als dir ist umbe mich".

Ich wil min lop keren

an wip die kunnen danken.

waz han ich von den überheren?

Das würde bedeuten, daß der Vortrag mit der starken Geste der Abkehr anfing, mit der der Sänger seinen früheren Dienst zurückzog. Wo das geschah, ob vor einer Gruppe von Damen oder vor einem gemischten Publikum, oder gar vor einer Männergruppe, liegt offen, d.h. er hatte sich von der jeweiligen Zusammensetzung des Publikums leiten lassen. Hie vor kann man einfach als Zeitangabe verstehen ("vorher", "früher", wie am Anfang einer anderen Strophe in diesem Lied, L 48, 12), oder als Ortsangabe hie plus vor den frowen. Allerdings bleibt die Unsicherheit, oder besser: die Möglichkeit verschiedener Inszenierungsweisen, auch dann, wenn die Strophe am Schluß steht, wie sie in den anderen zwei Hss. überliefert ist. Für den modernen Geschmack, der natürlich nicht unbedingt dem mittelalterlichen gleichzusetzen ist, gehört diese Geste der Zurückweisung, die vor allem den Damen gelten soll, nach der an eben diese Damen gerichteten programmatischen Strophe L 48, 38, sozusagen als Stichwort, bei dem der Sänger im Moment höchster dramatischer 
Wirkung buchstäblich abtreten kann.

Ein Lied, bei dem die handschriftliche Überlieferung weniger Spielraum bietet, ist das bekannte Mailied L 51, 13 Muget ir schouwen waz dem meien wunders ist beschert. In Hs. C besteht das Lied aus sechs Strophen, in Hs. A erscheinen vier Strophen, und zwar unter dem Namen eines anderen Minnesängers. Zwei Strophen finden sich auch unter den Carmina burana, zu denen das Lied in Ton und Form offensichtlich in einem nicht näher definierbaren Verhältnis steht. An der in C überlieferten Reihenfolge der Strophen ist aber nicht zu zweifeln, zumal das Lied eine klare dramatische Gestalt hat, mit einem Höhepunkt, einem plötzlichen Wechsel des Publikums, genau in der Mitte. Der Einschnitt ist so markant, daß frühe Herausgeber an zwei verschiedene Lieder gedacht haben; die drei ersten Strophen sollten ein Lied der niederen Minne bilden, die letzten seien der hohen Minne zuzuweisen. Natürlich sind heute auch diese Termini etwas suspekt geworden.

Man muß sich den Vortrag vor einer Frühlingslandschaft vorstellen, auf die der Sänger mit weit ausholenden Gesten hingedeutet haben könnte. In den ersten Strophen wendet er sich einem gemischten Publikum zu, dem er nicht nur Erscheinungen der Jahreszeit vor Augen hält, sondern auch die Gesellschaft selbst, in allen ihren Erscheinungsformen (pfaffen [...] leien). ${ }^{18}$ Eine Harmonie wird evoziert, die Natur und höfische Gesellschaft (keine dörperheit L 51, 24!) im Wir umfaßt. In der dritten Strophe scheint er sich dann ganz der Natur zuzuwenden, mit dem Wechsel vom ir der Zuhörer zum $d u$ scheint er eine intime Beziehung zum Vogel oder zum Grün des Mais vorzuzaubern. Teilweise vom Publikum abgewandt, betrachtet er die herrliche Landschaft und scheint sich in Gedanken zu verlieren. Die Blumen aber, die seinen Blick fesseln, rufen auch eine Erinnerung an die Farbe Rot wach, und der Minnesänger richtet seinen Blick und seine Worte plötzlich an einen bestimmten Brennpunkt in der Gesellschaft, an den roten Mund. Die neue Du-Anrede hatte vielleicht eine gewisse Spannung erregt, da sie jetzt eine bestimmte Frau betrifft, und diese Frau nicht gerade schmeichelhaft hervorhebt. Zunächst richtet der Sänger seine Aufmerksamkeit, und die seiner Zuhörer, sehr gezielt auf den Mund, der doch etwas Unpersönliches, ja

${ }^{18}$ In der Übersetzung Wapnewskis (s. Anm. 1, S. 59) geht allerdings die Möglichkeit einer Differenzierung des Publikums verloren: 'Seht alle Welt an...!' 
Unmenschliches an sich hat und der eigentlich für Disharmonie gesorgt hat. Hinter dem Du steckt vielleicht noch etwas von der Intimität der Beziehung zum Mai, die er gern auf die Beziehung zum roten Mund übertragen hätte. Natürlich braucht man wieder nicht an eine reale Reaktion seitens einer Hofdame zu denken, geschweige denn an ein Lächeln, das über den Sänger höhnen soll. Es gehörte zur Tradition, daß die schönen Augen oder der rote Mund einer Frau bei Männern Wunden verursachen konnten, einfach dadurch, daß die Frau sich in der Öffentlichkeit zeigte.

In den beiden letzten Strophen scheint der Sänger allmählich wieder zur Vernunft zu kommen. Er bleibt vor der Frau stehen, spricht sie aber mit ir an. Seine Wut schlägt sich noch in der fünften Strophe in Vorwürfen nieder (Z. 1-4), und mit der Frage wa nemt ir den muot? (Z. 5) begibt er sich an die Grenze zur Unhöflichkeit; dann beruft er sich etwas taktvoller auf ihre genaden, und in der letzten Strophe ist er wieder der bekannte wenn auch ungeduldige - Bittsteller. Er ist sich seiner Umwelt wieder bewußt, und wir sind es auch, denn ein Hinweis oder ein Blick des Sängers auf die herumstehende Gesellschaft ist vom Vortrag kaum wegzudenken, besonders in Z. 5-6; muget ir umbe sehen?/ sich fröit al diu welt gemeine. Was den Sänger zur Vernunft gebracht hat, ist teilweise die Anwesenheit des Publikums selbst, denn vom Minnesänger erwartete man ja, daß er die Stimmung am Hofe nicht verdarb, daß er sogar zur höfischen Freude beitrug. Um so dringender wird dann seine Bitte in der sechsten Strophe, da er durch seinen Zornausbruch auffällt. Ferner, mit seinem Mißmut stört er auch die Harmonie der Jahreszeit ( $\mathrm{Z}$. 2) und riskiert, daß der Mai an ihm vorübergeht, ohne daß er an den Freuden teilnimmt, die sie alle umgeben (vgl. auch Strophe 4, Z. 6: Owe so verlorner stunde...). Das ganze Lied ist also von seinen Vortragsverhältnissen geprägt, man spürt noch eine Interaktion zwischen Sänger, Publikum und Jahreszeit, und den Druck, unter dem der Vortragende steht.

Ein interessantes Beispiel ist ein weniger bekanntes Lied, L 42, 15 Swer verholne sorge trage:

1 Wil ab iemen wesen fro, daz wir in den sorgen iemer niht enleben? 
We wie tuont die jungen so

die von fröiden solten in den lüften sweben?

Ichn weiz anders weme ichz wizen sol, wan den richen wize ichz und den jungen.

die sint unbetwungen:

des stat in truren übel und stüende in fröide wol.

2 Wie fro Saelde kleiden kan,

daz si mir git kumber unde hohen muot!

So gits einem richen man

ungemüete: owe waz sol dem selben guot?

Min frou Saelde, wie si sich vergaz,

daz si mir sin guot ze minem muote

niene schriet, si guote!

min kumber stüende im dort bi sinen sorgen baz.

3. Swer verholne sorge trage,

der gedenke an guotiu wip, er wirt erlost;

Und gedenke an liehte tage:

die gedanke waren ie min bester trost.

Gegen den vinstern tagen han ich not

wan daz ich mich rihte nach der heide,

diu sich schamt vor leide:

so si den walt siht gruonen, so wirts iemer rot.

4 Frowe, als ich gedenke an dich, waz din reiner lip erwelter tugende pfliget:

So la stan! du rüerest mich

mitten an daz herze, da diu liebe liget.

Lieb und lieber des enmein ich niht,

ez ist aller liebest, daz ich meine.

du bist mir alleine

vor al der welte, frowe, swaz joch mir geschiht.

Das Lied besteht aus vier Strophen, von denen drei keine Beziehung zu irgendeinem Gegenüber verraten, eine Strophe aber eine Du-Anrede enthält. In der Ausgabe Friedrich Maurers erscheint diese Strophe als 
letzte, obwohl alle Hss. anders ordnen. Wie realitätsfremd die Strophenfolge der Ausgaben ist, läßt sich an einem Vergleich mit den Handschriften erkennen:

\begin{tabular}{lccccc} 
Maurer & Lachmann & \multicolumn{4}{c}{ Handschriften } \\
& & B & C & E & Ux \\
1 Wil ab & 3 & 2 & 2 & 1 & 1 \\
2 Wie fro & 4 & 4 & 4 & 2 & 2 \\
3 Swer & 1 & 1 & 1 & 4 & 4 \\
4 Frowe & 2 & 3 & 3 & 3 & 3
\end{tabular}

Was die Reihenfolge betrifft, bilden Hss. B und C eine Einheit, E und Ux eine zweite. Ferner, die Strophe, in der der Sänger seine frowe anspricht (= Maurers Nr. 4) erscheint tatsächlich überall als dritte; ja, nur in diesem Punkt sind alle Hss. einig. Für den mittelalterlichen Vortragenden also bildete die direkte Anrede keinen angemessenen Schluß des Liedes. Handelt es sich aber um eine echte Anrede? Zwar verwendet der Sänger das Pronomen $d u$ und nennt sein Gegenüber mit dem Adelstitel frowe, aber er spricht auch von gedenken, d.h. von der Erinnerung an sie als erprobtes Mittel gegen Sorgen, wobei er natürlich entweder an seinen eigenen Rat (Swer verholne sorge trage/der gedenke an guotiu wip) anknüpft (in der Strophenfolge von B/C) oder diesen Rat vorwegnimmt (in der Strophenfolge von E/Ux). Wenn das Lied in der Reihenfolge von $\mathrm{B} / \mathrm{C}$ gesungen wurde und mit dem allgemeinen Rat anfing, brauchte sich der Sänger bei Frowe, als ich gedenke an dich nicht unbedingt vor eine bestimmte Dame zu stellen, da er doch nur ein Erinnerungsbild anredete. Wollte er aber durch direkte Anrede die Scheinwerfer auf eine bestimmmte Frau richten, hätte er sie zum Gegenüber in Strophe 4 machen können, während er ihr erklärte, wie er sich sonst (d.h. wenn sie nicht vor ihm stand) über seine Sorgen hinweghalf, indem er an sie dachte; in Strophe 3 wandte er sich erst dann wieder dem breiteren Publikum zu, um anderen den selben Rat zu empfehlen. Diese Inszenierung bewahren die Hss. $\mathrm{E} / \mathrm{Ux}$.

Zum Schluß sei kurz auf ein paar Lieder hingewiesen, die keine deutlichen Beweise für Bewegungen oder Gesten seitens des Sängers bieten, die aber an die Unmittelbarkeit des realen Vortrags denken lassen. 
Walthers sog. Preislied Ir sult sprechen willekomen (L 56, 14), vielleicht 1203 am Wiener Hof gesungen, richtet sich an die großen Herren, von denen sich Walther ein Engagement als Minnesänger erhofft. Nach den fünf Strophen, die in drei bzw. vier Hss. überliefert sind, kommt in nur einer Hs. eine Zusatzstrophe (L 57, 15) vor, die nicht gut zu dem feierlichen Ton der anderen paßt: das Lied geht plötzlich in eine persönliche Minneklage über, wie man sie bei Walther und anderen Minnesängern dutzendweise findet:

6 Der ich vil gedienet han und ie mere gerne dienen wil, diust von mir vil unerlan, iedoch tuot si leides mir so vil.

Si kan mir verseren herze und den muot. nu vergebez ir got dazs an mir missetuot! her nach mac si sichs bekeren.

Während er aber die anderen Strophen vortrug, hat sich der Sänger wohl zwischen Männer- und Frauengruppen bewegt und dabei entsprechend Lob erteilt: in Strophe 5 (L 57, 7) preist er tiusche man, in 2 (L 56, 22) und 4 (L 56, 38) tiuschiu wip; auch andere Gruppen wie hoher bzw. niederer Adel wären für die anderen Strophen denkbar). Nach Strophe $4(56,38)$, in der er den Damen Lob spendet und vor ihnen steht, hätte er diese Einzelstrophe der Klage bereit haben können, die er bei passender Gelegenheit dem Vortrag hinzufügen könnte, falls eine bestimmte Dame unter der Frauengruppe war. In der einzigen Hs., die die Strophe enthält, folgt sie auf L 56, 38.

Das bekannte Lied L 50, 19 Bin ich dir unmaere, das die Minne als eine Gemeinschaft von zwei Herzen feiert, ist ein reines Anredelied, d.h. alle Strophen richten sich an ein Gegenüber. Hs. B hat nur zwei Strophen, die erste und die vierte, letztere aber an erster Stelle, so daß das Lied mit der Hervorhebung der frowe beginnt. In Hs. s beginnt der Sänger vielleicht vor einer Damengruppe (Strophe 3), die er forschend betrachtet und auf die er dann gelegentlich auch hindeutet (Z. 5-7), bevor er sich auf die Einzelne konzentriert; es folgen dann in s nur noch Strophe 2, in der der 
Sänger reagiert, als hätte die Dame seinen Blick nicht erwidert, und Strophe 4. Die Version in Hs. E hat vier Strophen, darunter als einzige die Zusatzstrophe L 176, 1 mit dem schwer verständlichen letzten Vers:

\author{
Si beginnent alle \\ miner frouwen füeze nemen war. \\ Mitten in dem schalle \\ so sich, frouwe, ouch under wilen dar. \\ Umbe die merkaere \\ la dir sin unmaere: \\ den grif ich wol naher baz: \\ daz versuoche alrerst, so denne daz.
}

Dadurch aber, daß Hs. E Strophe 3 nicht hat, würde sie die Anspielung auf die anderen in der Gesellschaft (das $s i$ der dritten Person pluralis) verlieren, wenn es diese Zusatzstrophe nicht gäbe. Kennzeichnend für das Lied ist doch, daß es eine Gemeinschaft von zwei Herzen propagiert und gegen die Außenwelt des Hofes abgrenzt, was wohl mit Gesten oder mit Blicken auf die anderen, die Außenstehenden, untermauert worden wäre. 